\title{
Asociatividad empresarial: estrategia para la competitividad de las PYMES en el Ecuador
}

\author{
Fecha de recepción: 12/03/2020 • Fecha de aceptación: 27/04/2020 • Fecha de publicación:10/06/2020
}

Fernando Gustavo Pérez Sisa

Universidad Central del Ecuador

fjperez@uce.edu.ec

https://orcid.org/0000-0002-6279-4366

\section{Resumen}

La asociatividad empresarial surge en base a la necesidad de crecimiento, desarrollo y oportunidades de las pequeñas y medianas empresas frente a un mundo globalizado en el cual cada día resulta más difícil mantener la competitividad y desarrollo sostenible de las mismas. Con la globalización en marcha se forjan grandes integraciones económicas, sociales y políticas; no obstante, dicha apertura internacional provoca desajustes macroeconómicos, lo cual se ve plasmado en mayor magnitud en el ámbito industrial.

A pesar de las dificultades que han experimentado las PYMES y que la asociatividad ha resultado ser una alternativa importante para mantenerse activas, es importante que los gobiernos estimulen su desarrollo a través de distintos mecanismos. Estas pueden ser leyes de protección, políticas de fomento, particularmente en las pequeñas y medianas empresas, donde se identifica un escaso nivel de competitividad para poder operar en las nuevas condiciones. Con la presente investigación se identificará como la asociatividad en otros países funciona de manera responsable y estratégica, sirviendo como eje de investigaciones para que el Ecuador se adapte a la globalización, mediante una investigación empírico documental, aportando diversas fuentes electrónicas y bibliográficas, resaltando los puntos más relevantes e importantes de aplicar este Modelo para el Ecuador. 


\begin{abstract}
Business associations arise from the need for growth, development and opportunities for small and medium-sized enterprises in a globalized world where it is increasingly difficult to maintain their competitiveness and sustainable development. With globalization underway, great economic, social and political integrations are being forged; however, this international openness causes macroeconomic imbalances, which are reflected in greater magnitude in the industrial field.

Despite the difficulties experienced by SMEs and the fact that associativity has proved to be an important alternative to remain active, it is important that governments stimulate their development through different mechanisms. These can be protection laws, promotion policies, particularly in small and medium-sized enterprises, where a low level of competitiveness is identified in order to operate under the new conditions. With this research, we will identify how partnership in other countries works in a responsible and strategic way, serving as the axis of research for Ecuador to adapt to globalization, through empirical documentary research, providing various electronic and bibliographic sources, highlighting the most relevant and important points to apply this model for Ecuador.
\end{abstract}

KEYWORDS: partnership, SMEs, competitiveness, innovation 


\section{Introducción}

El ser una empresa enmarcada dentro del ámbito de las PYMES (pequeña y mediana empresa) no es tarea fácil, debido a que poseen ciertas ventajas al momento de crearlas y tienen varios inconvenientes que se generan cuando ya se encuentra en marcha. Tanto pequeñas como medianas empresas miran como volverse más competitivas frente a empresas grandes misma tarea que es cada vez más compleja, pues las oportunidades para contar con información clara y oportuna se reducen notablemente.

Con la globalización en auge se vislumbra una continua y creciente integración de las economías, situación que provoca nuevos patrones de consumo, donde ultimadamente se ha generado una preocupación constante por la preservación de los recursos naturales. Ello ha conllevado a que las empresas busquen nuevos mercados, mostrando una nueva cara entorno a la competitividad empresarial, pues constantemente deben cambiar sus tácticas de modo que cumplan con las exigencias de los clientes.

Así, en este ámbito globalizado, por una parte, se identifica que las grandes empresas logran establecer estrategias para competir con empresas internacionales que ingresen al mercado nacional, pero, por otro lado, se puede resaltar la afectación que tienen las PYMES para poder cubrir sus necesidades dentro de su propio mercado, pues los costos para lograrlo son cada vez más altos.

A nivel nacional e internacional se puede identificar que las PYMES no logran tener un entorno adecuado, pues no logran incluir nuevas tecnologías dentro de sus procesos haciéndoles más difícil plantear innovaciones que les permitan crecer; de ahí que muchas empresas no duren tanto tiempo en el mercado y se empiecen a mostrar en quiebra.

En base a lo expresado anteriormente, es necesario implementar medidas para ayudar a las empresas medianas y pequeñas en su nivel o estándar de productividad, mejorando la competitividad nacional e internacional. Por eso se plantea la estrategia de generar asociatividad entre compañías similares. Este tipo de cooperaciones, en las cuales se mantiene una confianza recíproca y el compromiso de cada uno de los integrantes para el logro de un fin común, multiplicando las oportunidades de mantenerse activas en el mercado y de crecer con altos niveles de productividad.

Además, considerando que las PYMES se ven inmersas en mayor riesgo al momento de ingresar a un mundo globalizado, donde existe mayor exigencia en torno a la competitividad empresarial, es imprescindible analizar el comportamiento que este tipo de empresas han mostrado para mantenerse activas. Es ahí donde las asociatividades muestran su gran potencial, pues les permite obtener resultados admirables ya que la calidad de su producto mejora y les permite potenciar su competitividad en todos los niveles.

En el Ecuador durante el año 2017, se identificaron 802.709 microempresas representando el $90,78 \%$ el total de empresas legalmente registradas, mientras que las medianas y grandes empresas registraron un total de 81.614 organizaciones con un aporte del $9,23 \%$ de la capacidad empresarial según el Instituto Ecuatoriano de Estadística y Censo (INEC). 
Debido a estos datos apoyar a las microempresas se convierte en una labor fundamental para el desarrollo económico en el Ecuador, y la asociatividad aportara con grandes herramientas y estrategias que apoyaran al crecimiento y desarrollo industrial del Ecuador frente a los grandes cambios que genera la globalización.

\section{Asociatividad empresarial}

La temática sobre asociatividad constituye un aspecto que ha sido abordado desde diversas ópticas, pero que confluyen en algo en común, ser una estrategia para las pequeñas y medianas empresas.

Cuando se estudia el tema de asociatividad, existen algunos autores que no solamente lo plantean entorno a las empresas, sino que llevan al ámbito territorial. Uno de estos es Zoilo Pallares (2006), quien lo define como aquella cooperación que se genera entre los agentes locales cuyo objetivo es obtener un desarrollo sostenible, de modo que el territorio se vincule competitivamente a los movimientos del mundo (Pallares, 2006).

Si nos enfocamos en la asociatividad empresarial, una de las conclusiones más utilizadas e importantes a nivel internacional es la desarrollada por Rosales, quien da a conocer que es "un mecanismo de cooperación empresarial en el que cada una de las empresas que participan, mantienen su independencia jurídica y su autonomía gerencial, decidiendo voluntariamente su participación en un esfuerzo conjunto con los otros participantes para la búsqueda de un objetivo común" (Rosales, 1997).

Según Godínez en su libro redes empresariales, la gran diferenciación que existe entre agentes económicos torna importante la necesidad de generar alguna ventaja competitiva que permita mantenerse en el mercado. Ello conlleva a que sea preciso generar nexos horizontales, verticales y transversales entre empresas que sobrepasen el nivel de compra y venta que pueda generarse entre las mismas (Godínez, 2000).

Si bien, varios autores plasman el aspecto de asociatividad con ciertos puntos que sobresalen unos de otros, se puede evidenciar que en general todos muestran que la asociatividad empresarial constituye aquella cooperación que se genera entre empresas de modo que alcancen objetivos en común, los cuales están ligados a mostrar una mejor gestión, trayendo consigo incremento de la productividad y de la competitividad para los agentes involucrados (Mathews, 2014).

Sin embargo, la literatura existente muestra que no solo se esboza como tal a la asociatividad, sino que lo avizora como el manejo de redes empresariales en busca de un fin. Uno de los investigadores que plasma una definición sobre este tipo de redes es Carlos López (2003), quien da a conocer que las redes empresariales forman "una alianza importante y permanente entre un grupo limitado y claramente definido de empresas independientes, que colaboran para alcanzar objetivos comunes de mediano y largo plazo, orientados hacia el desarrollo de la competitividad de los distintos participantes". 
Un elemento a destacar es que este tipo de relaciones empresariales no se generan como un único modelo, sino que existen varias tipologías que se aplican según sea el objetivo a alcanzar. Existen cuatro grupos importantes en los cuales se puede clasificar; estos son (López, 2003):

- Redes o grupos empresariales: Constituyen formas asociativas empresariales, en las cuales se comparte información para generar competitividad en los sistemas productivos que desarrollan; de este modo, suman capacidades para alcanzar una efectividad colectiva.

- Redes verticales: Son aquellos procesos de encadenamiento entre los grandes productores y los pequeños clientes o potenciales distribuidores; o también como aquella relación que existe entre los clientes de grandes empresas y los proveedores de pequeñas empresas.

- Redes horizontales: Es aquel conjunto de empresas, máximo 20 empresas, que se encuentran en la misma rama productiva y localidad, las cuales se asocian para generar economías de escala reduciendo costos e incorporando la última tecnología del mercado.

- Clúster: Es aquel sector donde se identifica una alta concentración de productores, proveedores, y comercializadores enfocados en la misma rama industrial, pero que se interconectan por una actividad productiva individual que permite alentar la competitividad de las empresas ligadas al mismo sector productivo.

\section{Metodología}

La presente investigación se plasma a través de un diseño empírico documental, la información se adquiere mediante diversas fuentes electrónicas y bibliográficas, pretendiendo resaltar los puntos más relevantes e importantes de aplicar la asociatividad empresarial como una herramienta de crecimiento en las pequeñas y medianas empresas

La investigación se basó en evidencias obtenidas a través del análisis y recopilación de información proveniente de distintas fuentes electrónicas e impresas pretendiendo obtener datos relevantes acerca del tema. Enfatizando un enfoque cuantitativo, debido a que se analizan datos estadísticos, mismos que se obtienen de datos y resultados de organismos públicos como privados, por ejemplo, la Comunidad Andina de Naciones (CAN), textos y guías especializados en administración.

\section{Resultados}

\section{Pequeñas y medianas empresas - PYMES}

La representación de la sociedad mercantil ha incrementado su importancia en torno al ámbito económico; de este modo mientras adquiere un mayor protagonismo, se incrementará las formas y las actividades a las cuales tiene acceso, considerando también que se la complejidad se va incrementando a la par. 
Una definición amplia de lo que constituye una empresa estaría dada por la unión personas y recursos que buscan la consecución de un beneficio económico o social con el desarrollo de una actividad en particular (Galán, 2015).

Mientras que la Ley de Compañías del Ecuador (1999), en su artículo 1, las define como lo siguiente: "Contrato de compañía es aquél por el cual dos o más personas unen sus capitales o industrias, para emprender en operaciones mercantiles y participar de sus utilidades".

Siendo la empresa aquella entidad encargada de proveer de bienes y servicios, mediante el uso de los medios de producción, asumiendo ciertos riesgos inherentes de acuerdo al nivel de responsabilidad; es imperante manifestar que estos conflictos no son iguales para todas, y eso se debe en gran medida al tamaño que éstas tengan.

La Comunidad Andina de Naciones (2008), a través de su programa estadístico comunitario, establece las estadísticas comunitarias de las PYMES; así, a través de la Decisión 702, artículo 2, puntualiza que las PYMES son aquellas que aportan a la seguridad social y cuentan con registros contables, tomando en cuenta los distintos rangos en los que se divide

Estos umbrales que establece la CAN (2008), en el mismo Decreto 702 en su artículo 3 menciona que van enmarcados al personal y el valor bruto de ventas anuales, tal como se identifica en la Tabla 1.

Tabla 1.

Variables para clasificación de empresas

\begin{tabular}{lll}
\hline Variables & $\begin{array}{l}\text { Valor bruto de ventas anuales } \\
\text { (dólares) }\end{array}$ & Personal ocupado \\
\hline Estrato I & Menor a 100.000 & 1 a 9 \\
\hline Estrato II & De 100.001 a 1'000.000 & 10 a 49 \\
\hline Estrato III & De 1'000.001 a 2'000.000 & 50 a 99 \\
\hline Estrato IV & De 2'000.001 en adelante & 100 en adelante \\
\hline
\end{tabular}

Fuente: CAN, C. A. (2008)

Si bien, se establece dicha clasificación internacional, es competencia de cada país, determinar los parámetros para clasificarlas. En este sentido, en el Ecuador, a través de la Superintendencia de Compañías, se adopta una clasificación un tanto similar a la de la CAN, pero con cierta modificación en torno a las empresas medianas (Tabla 2). Cabe indicar que la clasificación se prioriza las ventas antes que el personal ocupado. 
Tabla 2.

Clasificación de empresas en el Ecuador

\begin{tabular}{lll}
\hline $\begin{array}{l}\text { Clasificación de las } \\
\text { empresas }\end{array}$ & $\begin{array}{l}\text { Volúmenes de ventas anuales } \\
\text { (dólares) }\end{array}$ & $\begin{array}{l}\text { Personal } \\
\text { ocupado }\end{array}$ \\
\hline Micro Empresa & Menor a 100.000 & 1 a 9 \\
\hline Pequeña Empresa & De 100.001 a 1'000.000 49 \\
\hline Mediana Empresa & De 1'000.001 a 5'000.000 & 50 a 199 \\
\hline Grande Empresa & De 5'000.001 en adelante & $\begin{array}{l}200 \text { en } \\
\text { adelante }\end{array}$ \\
\hline
\end{tabular}

Fuente: CAN, C. A. (2008)

Es generalizado, que el estudio respecto a las empresas se haya enfocado a aquellas que son de gran tamaño; no obstante, las PYMES han mostrado tener gran importancia dentro del desarrollo de los países, pues se han vuelto un elemento importante dentro de las políticas que se establecen.

Con el auge de las PYMES dentro del mundo globalizado, es importante tomar en cuenta que a pesar de tener criterios respecto a ventas y empleo que las definen, existen otros parámetros que permiten identificarlas.

Estos parámetros según el autor Urbano (2011) en su obra invitación al emprendimiento se detallan más profundamente a continuación:

- Cuentan con mayor innovación, tratando de generar productos personalizados, de modo que logren diferenciarse.

- Falta de acceso a financiamiento de largo plazo, lo cual les dificulta tener una planificación más amplia, y el manejo financiero se maneje a corto tiempo.

- Los costos de los servicios les resultan elevados respecto a los ingresos que obtienen, de ahí que no puedan acceder a última tecnología o cuenten con sistemas aseguradores de calidad.

- Existencia de competencia desleal, dado que no tienen acceso a toda la información, como a la que acceden las empresas grandes.

- Carga impositiva alta, lo cual resulta en incremento de los costos de la empresa.

- Falta de capacitación, dado que este tipo de empresas no cuenta con recursos que les permita invertir en capacitación para su personal.

Una vez teniendo claro el panorama sobre el que se trata las PYMES, es importante identificar 
como estas pueden optar por la estrategia de asociatividad de manera que se logren mantener en el tiempo.

\section{Asociatividad y PYMES}

Dada la exigencia de los mercados a nivel internacional, las empresas pequeñas y medianas no logran ingresar a los mismos, pues cuentan con una capacidad productiva pequeña dificultándole satisfacer la demanda de los potenciales clientes. Es ahí, donde la actividad asociativa constituye una propuesta atractiva para las PYMES, debido a que les brinda un aporte competitivo frente a los grandes desafíos de los mercados internacionales.

Como lo plantea Coase en su obra cuarenta años de un artículo, "la asociatividad como forma organizacional en la empresa, se origina a partir de la necesidad de reducir los costos de transacción para repartir los costos asociados a la gestión del intercambio y el desarrollo en economías de escala" (Figueras, 2001).

Las estrategias colectivas son pilares importantes para lograr estándares y niveles de productividad que aportaran a la competitividad en las empresas medianas y pequeñas; buscando igualar o competir con a las grandes organizaciones.

Al ser un aspecto importante la manera de asociarse de las pequeñas y medianas empresas, según abril y Tono (2010) se debe tomar en cuenta que existen diferentes etapas, tales como:-

- Etapa de gestación: Se inician con los acercamientos entre las empresas interesadas que deben tener características similares. De este modo, se identifican los objetivos comunes y se establece el rol que desempeñara cada empresa.

- Etapa de estructuración: es este periodo se determina las estrategias que se van a generar de modo que se alcance las metas establecidas.

- Etapa de madurez: En ésta se busca que el grupo alcance la autosostenibilidad.

- Etapa productiva: se determina el bosquejo del proyecto para el cumplimiento de las metas asignadas.

- Etapa de declinación: Se debe tomar la decisión de seguir o no con el grupo, ya que las metas planteadas inicialmente ya no resultan positivas y por el contrario empiezan a volverse en contra de la empresa; casi siempre va ligada con la disminución de la cooperación.

Como se planteó previamente, existen distintos tipos de asociatividad por los cuales pueden optar las PYMES; sin embargo, es importante tomar en consideración lo explicado por Dini, Ferrano \& Gasaly (2007):

- Las redes deben propender a contar con pocos integrantes, dado que a mayor cantidad de integrantes mayor será el costo de transacción que las empresas deben cubrir respecto al tema 
de confianza y labor colectiva. Las excepciones que se pueden dar para tener un alto número de integrantes serán en base a la cultura y capacidad asociativa que se posea.

- En los clusters los beneficiarios son todos los individuos o emprendimientos involucrados al mismo sin distinción. Es decir, si una empresa se encuentra en la localidad en la que se generará una asociatividad de varias empresas y se enfoca en el desarrollo de actividades en común, esta podría convertirse en parte del clúster.

\section{Experiencias internacionales en la Asociatividad de las PYMES}

Existen varios casos relacionados a proyectos de articulación productiva cuyo principal objetivo era alcanzar y mejorar la productividad de las PYMES, para lo cual buscaron usaron varias formas de integración productiva, entre las que se encuentra la asociatividad.

A continuación, se plasma algunas de las experiencias internacionales, basado en Dini, Ferraro \& Gasaly (2007):

- México: La entidad coordinadora de Fomento al Comercio Exterior del Estado de Guanajuato, en el año 2003 propicio el proyecto que buscaba mejorar la productividad de las MIPYMES del Estado de Guanajuato. Para ello, tanto en el sector de cuero y calzado; así como en proveeduría textil y de cerámica decorativa, estableció actividades colectivas que permitan fortalecer la innovación de productos y proceso, a través de cadenas de valor y la articulación de las empresas que pertenecían a dichos sectores. Como resultado, se logró la continuidad de las empresas en el mercado y el desarrollo comercial en el área.

- Nicaragua: La Organización de las Naciones Unidas para el Desarrollo Industrial en colaboración con la Cleaner Production Centres en el año 2005, establecieron la idea de desarrollar la competitividad de once clusters enfocados a las áreas de artesanía, turismo y agropecuaria, éste último con particular atención en la producción de leche, cultivos de cacao, cultivo de plátanos. Los resultados que se alcanzaron fueron establecimiento de cooperativas lácteas, generación de programas de infraestructura rural y capacitación de las instituciones locales.

- Colombia: Constituye un proyecto generado en el año 2019 por el grupo de investigadores encabezada por la Ing. Amanda Vargas para establecer el clúster en la cadena de producción en los pequeños productores de papa criolla. Se logró asociar a los integrantes del municipio de Subacoche, ocasionando que puedan introducirse y cubrir las necesidades de mercados más exigentes, especializándose en la estandarización de la información y mejora en la cadena de valor agregado (Vargas, 2019).

- Perú: En el año 2004 se crea las redes de empresas de confecciones con el apoyo del Centro de Promoción de la Pequeña y Microempresa. El objetivo de esta asociatividad era desarrollar la competitividad de las PYMES de confecciones de Gamarra - Lima y de turismo de Cuzco. Con la puesta en marcha de estas redes empresariales, se facilitó los trámites legales y se generaron acuerdos de colaboración productivas con empresas grandes, de modo que se promocionen sus productos. 
- Uruguay: La Asociación de bodegas exportadoras de vinos finos de Uruguay plantea el proyecto que reuniría a las principales empresas vitivinícolas uruguayas, que son conocidas por ser exportadoras de vinos finos, en el año 2003. Esto provocó que los vinos finos de Uruguay logren alcanzar competitividad en el mercado internacional.

- España: En este país se observa la interacción de PYMES que se orientan a la exportación. Así, se ha establecido una estructura común con la existencia de un solo gerente; el cual este encargado de atender las necesidades de los negocios involucrados. Cabe indicar que en este tipo de asociatividad se encuentra inmerso el Estado, el mismo que entrega un tipo de subsidio (Liendo \& Martinez, 2001).

Como se observa en los ejemplos planteados, sin importar el tipo de asociatividad que se establezca, se logran alcanzar importantes resultados en dos puntos importantes: la innovación y el acceso a mercados. Con ello, se logra una mayor competitividad de las empresas involucradas y además se logra que se mantengan en el mercado.

Las PYMES constituyen un elemento importante en el desarrollo social y económico de los países, pues desde sus trincheras generan riqueza y empleo. No obstante, deben afrontar la competencia de las grandes empresas, las cuales cuentan con un mayor poder económico.

El crecimiento empresarial en el Ecuador, se lo detalla en el siguiente cuadro (Tabla 3), donde posteriormente se analizará el impacto que poseen para el desarrollo económico:

Tabla 3.

Evolución y estructura empresarial en el Ecuador según su tamaño

\begin{tabular}{lllllll}
\hline Empresas Activamente Registradas Año 2017 & & & & \\
\hline & $\begin{array}{l}\text { Empresas } \\
\text { Tamaño }\end{array}$ & $\begin{array}{l}\text { Porcentaje } \\
\text { Registradas }\end{array}$ & $\begin{array}{l}\text { Ventas } \\
\text { Tamaño }\end{array}$ & $\begin{array}{l}\text { Pegistradas } \\
\text { Ventas }\end{array}$ & $\begin{array}{l}\text { Impacto } \\
\text { Laboral }\end{array}$ & $\begin{array}{l}\text { Porcentaje } \\
\text { de Afiliados }\end{array}$ \\
\hline Microempresas & 802.709 & $90,78 \%$ & $\$ 1.035,63$ & $0,96 \%$ & 720.449 & $24,51 \%$ \\
\hline $\begin{array}{l}\text { Pequeñas y } \\
\text { Medianas }\end{array}$ & 77.547 & $8,77 \%$ & $\$ 29.817,48$ & $27,64 \%$ & 1077.588 & $36,66 \%$ \\
\hline $\begin{array}{l}\text { Grandes } \\
\text { Empresas }\end{array}$ & 4.067 & $0,46 \%$ & $\$ 77.024,89$ & $71,40 \%$ & 1141.373 & $38,83 \%$ \\
\hline TOTAL & 884.324 & $100 \%$ & $\$ 107.878,00$ & $100 \%$ & 2939.410 & $100 \%$ \\
\hline
\end{tabular}

Fuente: Instituto Nacional de Estadística y Censo (INEC Ecuador)

En el Ecuador, al año 2017, al mirar el sector formal de la economía, se identifica que las microempresas representan el $90,78 \%$; las pequeñas y medianas empresas equivalen el $8,77 \%$, y las grandes empresas representan el $0,46 \%$. Si se compara estos datos con los últimos cinco años, donde las PYMES representaban el 10,03\%, se puede identificar que éstas han ido en decremento (INEC, 2017).

Esto se corrobora al ver los datos a nivel de empleo donde las PYMES pasaron de cubrir el $39,94 \%$ del mercado en el 2012 , a $36,66 \%$ en el año 2017. No obstante, al verificar dichos datos 
en torno a las ventas que generan dichas empresas, se puede identificar que las PYMES para el año 2017, alcanza el 27,64\%; mientras que los ingresos registrados en el año 2012 llegan a cubrir el $26,71 \%$, un incremento mínimo.

Al llevar estos datos al ámbito territorial, se identifica que las PYMES se concentran en la provincia de Pichincha y Guayas, con una participación de 29,3\% y 25,5\% respectivamente. En el resto de las provincias del Ecuador, la participación que tienen no supera el $6 \%$ por cada una.

Estos datos muestran que las PYMES que se encuentran distribuidas en otras zonas distintas a la capital y a su puerto principal, no alcanzan la competitividad necesaria para alcanzar mercados internacionales. Es ahí donde aparecen las principales asociaciones que pueden darse entre estas empresas.

Un ejemplo de asociatividad dado en el Ecuador viene de la mano de los dos clusters que se dieron; el primero localizado en Atuntaqui enfocado al área textil y de confecciones; y el segundo creado en Ambato para el sector de cuero y calzado. Los alcances de estas relaciones empresariales fueron la implantación de una planificación estratégica, de tal manera que se logre incorporar nueva tecnología en las organizaciones productivas involucradas.

Otro de los grandes ejemplos que podemos ver en el Ecuador, es el cluster creado en el cantón Cuenca para el sector de Muebles. En este proyecto, una empresa grande que produce y comercializa muebles, vio imperante la necesidad de asociarse con pequeños artesanos para poder abastecer toda la producción nacional e internacional. Éste es un caso de consolidación de red de productores, lo cual mejoró los ingresos de los pequeños empresarios.

El sector de las joyas no se queda atrás, dada la cantidad de pequeñas empresas dedicadas a la joyería en Chordeleg, consideraron importante crear un cluster que fomente el desarrollo social y económico. Como resultado se obtuvo la generación de una marca diferenciada, la cual proveería al mercado nacional e internacional de joyas con diseños exclusivos trabajados en metales preciosos, volviéndolo un agente económico importante a nivel mundial. Así, cada pequeña empresa involucrada alcanzó nuevos conocimientos que facilitaron la mejoría de sus procesos productivos.

\section{Conclusiones}

Con la globalización en marcha se forjan grandes integraciones económicas, sociales y políticas; no obstante, dicha apertura internacional provoca desajustes macroeconómicos, los cual se ve plasmado en mayor magnitud en el ámbito industrial, particularmente en las pequeñas y medianas empresas, donde se identifica un escaso nivel de competitividad para poder operar en las nuevas condiciones.

Si bien a nivel internacional se pueden identificar interacciones espontaneas que han dado como resultado concentraciones empresariales, esto no es suficiente, pues es necesario crear una base de confianza sólida que permita la real integración de todos los miembros tanto en conocimientos como en capacidades que dispone cada uno. Por ello, cuando se crea cualquier tipo de 
asociatividad es imprescindible que se establezcan reglas claras que fomenten la coordinación de la misma.

La tarea de generar la asociatividad de empresas es algo complicada, debido a que se debe empezar por eliminar los desequilibrios de conocimiento y barreras que existan entre todos los interesados en realizar la alianza, además de tener la predisposición para mantener una planificación a largo plazo. No obstante, esta constituye una gran estrategia para las pequeñas y medianas empresas, debido a que les permite eliminar la asimetría que existe en relación al capital que deban invertir y a la par les otorga mayor competitividad frente a las grandes empresas.

Además, el desarrollo de clusters para las PYMES es un elemento importante pues les facilita el desarrollo de nuevas capacidades dentro del nivel comercial y productivo, lo cual le genera un valor agregado respecto a los bienes y servicios que ofertan las empresas.

A pesar de presentar casos exitosos de asociatividad a nivel nacional e internacional; varios han sido los casos que a nivel de América Latina no han alcanzado el objetivo planteado. Esta situación ha sido provocada en gran medida al no conseguir el cooperativismo necesario y que, a pesar de estar asociados, las empresas actúan de manera individual dentro de su proceso productivo y de comercialización. Por lo tanto, es muy importante que a la par de crear la asociatividad, se promueva un gran nivel de confianza.

Finalmente, a pesar de las dificultades que han experimentado las PYMES y que la asociatividad ha resultado ser una alternativa importante para mantenerse activas, es importante que los gobiernos estimulen su desarrollo a través de distintos mecanismos. Estos pueden leyes de protección, políticas de fomento, creación de programas de financiamiento y programas de capacitación. 


\section{Referencias}

Abril, M. I., \& Tono, L. (2010). Propuesta de asociatividad de las PYMES de Cuenca como medio de fomentar exportaciones. Cuenca.

Bueno, E. (1974). Análisis críticos de los objetivos y subjetivos de la Empresa. Madrid.

CAN, C. A. (2008). Decisión 702. Lima, Perú.

Dini, M. (1997). Forjando Aglomeraciones en Chile y Centroamérica. Enseñanzas de la Experiencia. Reunio SELA. Lima, Perú.

Dini, M., Ferraro, C., \& Gasaly, C. (2007). PYMES y Articulación productiva. Resultados y lecciones a partir de experiencias en América Latina. Chile: CEPAL.

Ecuador, C. N. (1999). Ley de Compañías. Ecuador.

Figueras, A. J. (2001). Ronald Coase, a cuarenta años de un artículo. Actualidad Económica Año 1, Nº 51.

Galán, J. S. (12 de Diciembre de 2015). Empresa. Economipedia, 1. Obtenido de https://economipedia.com/ definiciones/empresa.html

INEC. (2017). Directorio de Empresas. Quito.

Liendo, M., \& Martinez, A. (2001). Asociatividad. Una alternativa para el desarrollo y cremiento de las PYMES. Argentina.

López, C. (2003). Redes empresariales. Experiencias en la Región Andina. Perú.

Mathews, J. C. (2014). Asociatividad empresarial. Agenda 2014. Propuestas para mejorar la Descentralización.

Pallares, Z. (2006). Desarrollo local y asociatividad territorial.

Rosales, R. (1997). La Asociatividad como estrategia de fortalecimiento de las PYMES. México: Universidad de Texas.

Urbano, D., \& Toledano, N. (2011). Invitación al emprendimiento: Una aproximación a la creación de empresas. Barcelona: UOC.

Vargas, A. (22 de Noviembre de 2019). La asociatividad para articular cadenas productivas en Colombia.El caso de los pequeños productores de papa criolla en Subachoque, Cundinamarca. Cooperativismo y DesarroIlo. Obtenido de http://198.46.134.239/index.php/co/article/view/2999 
